VOL. 57 (1998) [261-274]

\title{
CLASS NUMBERS OF REAL QUADRATIC FIELDS
}

\author{
JAE MOON KIM
}

Let $k=\mathbb{Q}(\sqrt{m})$ be a real quadratic field. It is well known that if 3 divides the class number of $k$, then 3 divides the class number of $\mathbb{Q}(\sqrt{-3 m})$, and thus it divides $B_{1, \chi \omega^{-1}}$, where $\chi$ and $\omega$ are characters belonging to the fields $k$ and $\mathbb{Q}(\sqrt{-3})$ respectively. In general, the main conjecture of Iwasawa theory implies that if an odd prime $p$ divides the class number of $k$, then $p$ divides $B_{1, \chi \omega^{-1}}$, where $\omega$ is the Teichmüller character for $p$.

The aim of this paper is to examine its converse when $p$ splits in $k$. Let $k_{\infty}$ be the $\mathbb{Z}_{p}$-extension of $k=k_{0}$ and $h_{n}$ be the class number of $k_{n}$, the $n$th layer of the $\mathbb{Z}_{p}$-extension. We shall prove that if $p \mid B_{1, \chi \omega^{-1}}$, then $p \mid h_{n}$ for all $n \geqslant 1$. In terms of Iwasawa theory, this amounts to saying that if $M_{\infty} / k_{\infty}$ is nontrivial, then $L_{\infty} / k_{\infty}$ is nontrivial, where $M_{\infty}$ and $L_{\infty}$ are the maximal abelian $p$-extensions unramified outside $p$ and unramified everywhere respectively.

\section{INTRODUCTION}

Fix a square free positive integer $m$ and let $k=\mathbb{Q}(\sqrt{m})$. Class numbers of these real quadratic fields have been studied for a long time. Two outstanding formulas related to class numbers are the analytic (classical or $p$-adic) class number formula [7] and the index theorem of circular units discovered by Sinnott [6].

In this paper we study the class number of $k$ by examining the $p$-divisibility of the class number for each prime $p$. When $p=2$, the answer is well known and can be easily checked either by considering the genus field of $k$ or by using cohomological arguments: if the discriminant of $k$ has at least three distinct prime divisors, then the class number is divisible by 2 . Note that the converse of this statement is not true. For instance, the class number of $\mathbb{Q}(\sqrt{85})$ is 2 and that of $\mathbb{Q}(\sqrt{21})$ is 1 . Both of these fields have discriminants with exactly two prime divisors.

The answer for $p=3$ is also known [5, 7]: if 3 divides the class number of $k$, then 3 divides the class number of $\mathbb{Q}(\sqrt{-3 m})$. This can be proved either by applying the $p$-adic class number formular or by using the Kummer pairing as Scholz did [5]. The

Received 31st July, 1997

This work was partially supported by the Basic Science Research Institute program, Ministry of Education, Korea, \#BSRI-96-1414, and was completed while the author was visiting the Ohio State University during the sabbatical year 1996-1997.

Copyright Clearance Centre, Inc. Serial-fee code: 0004-9729/98 \$A2.00+0.00. 
converse of this does not hold either. For example, the class number of $\mathbb{Q}(\sqrt{85})$ is 2 , but that of $\mathbb{Q}(\sqrt{-255})$ is 12 . Let $\chi$ be the nontrivial character belonging to $k$ and $\omega$ be the Teichmüller character for $p=3$. Then $\chi \omega^{-1}$ belongs to the field $\mathbb{Q}(\sqrt{-3 m})$ and $-B_{1, \chi \omega^{-1}}$ is the class number of $\mathbb{Q}(\sqrt{-3 m})$. Thus we can rephrase the statement as 3 divides $B_{1, \chi \omega^{-1}}$ if 3 divides the class number of $k$.

This can be generalised to an arbitrary odd prime $p$ by using the main conjecture of Iwasawa theory. Let $\omega$ be the Teichmüller character for $p$. Let $k_{\infty}$ be the $\mathbb{Z}_{p^{-}}$ extension of $k$ and $M_{\infty}$ be its maximal abelian $p$-extension unramified outside $p$. Let $f_{\chi}$ be the Iwasawa power series in $\Lambda=\mathbb{Z}_{p}[[T]]$ corresponding to the $p$-adic $L$-function $L_{p}(s, \chi)$. Then by the main conjecture, which is a theorem now [4], $\mathrm{Gal}\left(M_{\infty} / k_{\infty}\right)$ is pseudo-isomorphic to $\Lambda /\left(f_{\chi}\right)$ as a $\Lambda$-module. We also have

$$
f_{\chi}(0)=L_{p}(0, \chi)=-B_{1, \chi \omega^{-1}}
$$

Thus if $p$ does not divide $B_{1, \chi \omega^{-1}}$, then $f_{\chi}$ is a unit in $\Lambda$. Hence Gal $\left(M_{\infty} / k_{\infty}\right)$ is trivial, since it has no nonzero finite $\Lambda$-submodules (see [3, appendix]). Therefore $\mathrm{Gal}\left(L_{\infty} / k_{\infty}\right)$ is also trivial, where $L_{\infty}$ is the maximal unramified Abelian $p$-extension of $k_{\infty}$. Thus $p$ does not divide the class number of $k$. To summarise, we proved:

THEOREM 1. Let $k=\mathbb{Q}(\sqrt{m})$ and $p$ be an odd prime. If $p$ divides the class number of $k$, then $p$ divides $B_{1, \chi \omega^{-1}}$.

The aim of this paper is to discuss the converse of theorem 1. According to the previous example for $p=3$, the converse cannot be true in general. However, we have the following result when $p$ splits in $k$. For each $n \geqslant 0$, let $h_{n}$ be the class number of $k_{n}$, the $n$th layer of the $\mathbb{Z}_{p}$-extension $k_{\infty}$ of $k$.

THEOREM 4. Suppose an odd prime $p$ splits in $k=\mathbb{Q}(\sqrt{m})$. If $p$ divides $B_{1, \chi \omega^{-1}}$, then $p$ divides $h_{n}$ for all $n \geqslant 1$.

For the proof of Theorem 4, we shall use circular units defined by Sinnott and his index Theorem [6]. In Section 2, we briefly review his definition of circular units and compute cohomology groups of them in the $\mathbb{Z}_{p}$-extension. In Section 3 , we shall assume that $p$ splits in $k$, so there are two prime ideals $\wp_{0}$ and $\widetilde{\varphi}_{0}$ above $p$ in $k$. Let $\wp_{n}$ and $\widetilde{\varphi}_{n}$ be the prime ideals of $k_{n}$ above $\wp_{0}$ and $\widetilde{\wp}_{0}$ respectively. We shall see that every circular unit $\delta_{n}$ of $k_{n}$ whose norm to $k_{0}$ equals 1 has the property that $\delta_{n}=\alpha_{n}^{\sigma-1}$ for some $\alpha_{n} \in k_{n}$ satisfying $\left(\alpha_{n}\right)=\wp_{n}^{g_{n}}{\widetilde{\vartheta_{n}}}^{\tilde{g}_{n}}$ for some integers $g_{n}$ and $\widetilde{g}_{n}$, where $\sigma$ is a topological generator of the Galois group $\Gamma=\mathrm{Gal}\left(k_{\infty} / k\right)$. Then we pick a special $\delta_{n}$ and show that

$$
g_{n}-\tilde{g}_{n} \equiv \pm \sqrt{d} B_{1, \chi \omega^{-1}} \bmod p \mathbb{Z}_{p}
$$

where $d$ is the conductor of $k$. Finally, we apply this congruence to prove Theorem 4 . 


\section{Circular Units}

Let $F$ be an abelian field. For each $n>2$, let $F_{n}=F \cap \mathbb{Q}\left(\zeta_{n}\right)$ and $C_{F_{n}}=$ $N_{\mathbb{Q}\left(\zeta_{n}\right) / F_{n}}\left(C_{\mathbb{Q}\left(\zeta_{n}\right)}\right)$, where $C_{\mathbb{Q}\left(\zeta_{n}\right)}$ is the group of the cyclotomic units of $\mathbb{Q}\left(\zeta_{n}\right)$ in the usual sense. We define the group of circular units $C_{F}$ of $F$ as the multiplicative subgroup of $F^{\times}$generated by $C_{F_{n}}$ together with -1 (see [6]). Note that if $n$ is prime to the conductor of $F$, then $F_{n}=\mathbb{Q}$ and so $C_{F_{n}}=\{1\}$. Thus there are only finitely many $n$ 's to be considered in the definition of $C_{F}$. For example, when $F=k=$ $\mathbb{Q}(\sqrt{m}), C_{k}=\left\langle N_{\mathbb{Q}\left(\zeta_{d}\right) / k}\left(C_{\mathbb{Q}\left(\zeta_{d}\right)}\right),-1\right\rangle$, where $d$ is the conductor of $k$ ( $d$ will always mean the conductor of $k$ throughout this paper). To see this, first observe that $k \cap$ $\mathbb{Q}\left(\zeta_{n}\right)$ is either $\mathbb{Q}$ or $k$. If $k \cap \mathbb{Q}\left(\zeta_{n}\right)=\mathbb{Q}$, then $C_{k_{n}}=\{1\}$. Otherwise, $\mathbb{Q}\left(\zeta_{n}\right)$ contains $\mathbb{Q}\left(\zeta_{d}\right)$ as a subfield and thus $N_{\mathbb{Q}\left(\zeta_{n}\right) / k}\left(C_{\mathbb{Q}\left(\zeta_{n}\right)}\right)$ is contained in $N_{\mathbb{Q}\left(\zeta_{d}\right) / k}\left(C_{\mathbb{Q}\left(\zeta_{d}\right)}\right)$.

Fix an odd prime $p$ with $(p, m)=1$, and let $k_{\infty}=\bigcup_{n \geqslant 0} k_{n}$ be the $\mathbb{Z}_{p}$-extension of $k=k_{0}=\mathbb{Q}(\sqrt{m})$. For each $n \geqslant 0$, we denote the group of circular units of $k_{n}$ by $C_{n}$. It is not hard to show that

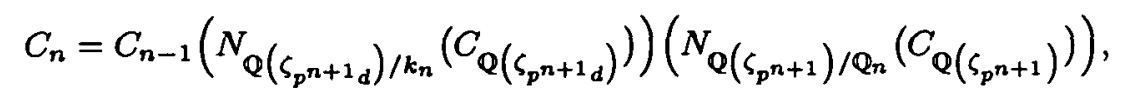

where $\mathbb{Q}_{n}$ is the subfield of $\mathbb{Q}\left(\zeta_{p^{n+1}}\right)$ whose degree over $\mathbb{Q}$ is $p^{n}$. Thus the generators of $C_{n}$ are given so explicitly that we can compute the cohomology groups of circular units in the $\mathbb{Z}_{p}$-extension. Another feature of the circular units is the following index theorem discovered by Sinnott [6].

THEOREM. Let $E_{n}$ be the unit group of $k_{n}$ and $h_{n}$ be the class number of $k_{n}$. Then $\left[E_{n}: C_{n}\right]=2^{c_{n}} h_{n}$ for some integer $c_{n}$.

Before we compute the cohomology groups of $C_{n}$, we set up some notation. For each integer $s \geqslant 1$, we choose a primitive $s$ th root $\zeta_{s}$ of 1 so that $\zeta_{t}^{t / s}=\zeta_{s}$ if $s \mid t$. Let $K=\mathbb{Q}\left(\zeta_{d}\right), F=\mathbb{Q}\left(\zeta_{p}\right)$ and $K^{\prime}=\mathbb{Q}\left(\zeta_{p d}\right)$. We denote their cyclotomic $\mathbb{Z}_{p}$-extensions by $K_{\infty}, F_{\infty}$, and $K_{\infty}^{\prime}$. Let $\sigma$ be the topological generator of the Galois group $\Gamma=\operatorname{Gal}\left(K_{\infty}^{\prime} / K^{\prime}\right)$ which maps $\zeta_{p^{n}}$ to $\zeta_{p^{n}}^{1+p}$ for all $n \geqslant 1$. Restrictions of $\sigma$ to various subfields are also denoted by $\sigma$. Let $\Delta=\mathrm{Gal}(K / k), \bar{\Delta}=$ $\operatorname{Gal}(K / \mathbb{Q})$ and $\Delta_{k}=\operatorname{Gal}(k / \mathbb{Q})=\{i d, \rho\}$. Elements of $\Delta$ or $\bar{\Delta}$ will be denoted by $\tau$ 's. Let $R$ be the set of all roots of 1 in $\mathbb{Z}_{p}$, that is, $R=\left\{\omega \in \mathbb{Z}_{p} \mid \omega^{p-1}=1\right\}$. Then $R$ can be regarded as the Galois group $\mathrm{Gal}(F / \mathbb{Q})$ or any Galois group isomorphic to it such as $\operatorname{Gal}\left(F_{n} / \mathbb{Q}_{n}\right)$. For $m>n$, let $G_{m, n}$ be the Galois group Gal $\left(K_{m}^{\prime} / K_{n}^{\prime}\right)$ and $N_{m, n}$ be the norm map $N_{K_{m}^{\prime} / K_{n}^{\prime}}$ from $K_{m}^{\prime}$ to $K_{n}^{\prime}$. We shall abbreviate $G_{m, 0}$ and $N_{m, 0}$ to $G_{m}$ and $N_{m}$ respectively. $G_{m, n}$ will also mean the Galois groups $\operatorname{Gal}\left(k_{m} / k_{n}\right), \operatorname{Gal}\left(F_{m} / F_{n}\right)$ and $\operatorname{Gal}\left(\mathbb{Q}_{m} / \mathbb{Q}_{n}\right)$. Similarly $N_{m, n}$ will have various meanings. Finally we fix a generator $\psi_{n}$ of the character group of $\operatorname{Gal}\left(\mathbb{Q}_{n} / \mathbb{Q}\right)$ 
such that $\psi_{n}(\sigma)=\zeta_{p^{n}}$. Thus $\psi_{n}$ is an even character of order $p^{n}$ with conductor $p^{n+1}$ such that $\psi_{n}^{p}=\psi_{n-1}$.

THEOREM 2. Suppose $p$ splits in $k$. For $m>n \geqslant 0$, we have the following.

$$
\begin{aligned}
C_{m}^{G_{m, n}} & =C_{n}, \\
\widehat{H}^{0}\left(G_{m, n}, C_{m}\right) & \simeq \mathbb{Z} / p^{m-n} \mathbb{Z}, \\
\hat{H}^{-1}\left(G_{m, n}, C_{m}\right) & \simeq\left(\mathbb{Z} / p^{m-n} \mathbb{Z}\right)^{2} .
\end{aligned}
$$

Proof of (1): It is clear that $C_{n} \subset C_{m}^{G_{m, n}}$. To prove the converse, we invoke a theorem of Ennola on relations of cyclotomic units [1]: If a cyclotomic unit $\xi=$ $\prod_{1 \leqslant a<n}\left(1-\zeta_{n}^{a}\right)^{x_{a}}$ in $\mathbb{Q}\left(\zeta_{n}\right)$ is a root of 1 for some integers $x_{a}$, then $Y(\theta, \xi)=0$ for every even character $\theta$ of conductor $n$, where $Y(\theta, \xi)=\sum_{1 \leqslant a<n} \theta(a) x_{a}$.

To prove $C_{m}^{G m, n} \subset C_{n}$, it is enough to check the inclusion when $m=n+1$. Suppose that $u \in C_{n+1}$ is fixed by $G_{n+1, n}$, that is $u^{\sigma^{p^{n}}}=u$. By $(*), u=u_{n} v_{n+1}$ for some $u_{n} \in C_{n}$ and $v_{n+1} \in N_{K_{n+1}^{\prime} / k_{n+1}}\left(C_{K_{n+1}^{\prime}}\right) N_{F_{n+1} / \mathbb{Q}_{n+1}}\left(C_{F_{n+1}}\right)$. Since $u_{n}^{\sigma^{p^{n}}}=$ $u_{n}$, we have $v_{n+1}^{\sigma^{p^{n}}}=v_{n+1}$. Thus we may assume $u_{n}=1$. Then we can write $u$ as

$$
u=\prod_{\substack{0 \leqslant l<p^{n} \\ 0 \leqslant k<p}}\left(\prod_{\substack{\omega \in R \\ \tau \in \Delta}}\left(\zeta_{p^{n+2}}^{\sigma^{l+k p^{n}} \omega}-\zeta_{d}^{\tau}\right)^{a_{l, k}}\left(\zeta_{p^{n+2}}^{\sigma^{l+k p^{n}} \omega}-\zeta_{d}^{\rho \tau}\right)^{b_{l, k}} \prod_{\omega \in R}\left(\zeta_{p^{n+2}}^{\sigma^{l+k p^{n}} \omega}-1\right)^{c_{l, k}}\right),
$$

for some integers $a_{l, k}, b_{l, k}$ and $c_{l, k}$.

We apply Ennola's theorem to the relation $u^{\sigma^{p^{n}}-1}=1$ with characters of the form $\psi_{n+1}^{j} \chi$ for $0<j<p^{n+1},(j, p)=1$. Notice that

$$
\begin{gathered}
Y\left(\psi_{n+1}^{j} \chi, \prod_{\substack{\omega \in R \\
\tau \in \Delta}}\left(\zeta_{p^{n+2}}^{\sigma^{l+k p^{n}}{ }^{\prime}}-\zeta_{d}^{\tau}\right)\right)=(p-1) \frac{\phi(d)}{2} \psi_{n+1}^{j}\left(d \sigma^{l+k p^{n}}\right) \\
Y\left(\psi_{n+1}^{j} \chi, \prod_{\substack{\omega \in R \\
\tau \in \Delta}}\left(\zeta_{p^{n+2}}^{\sigma^{l+k p^{n}} \omega}-\zeta_{d}^{\rho \tau}\right)\right)=-(p-1) \frac{\phi(d)}{2} \psi_{n+1}^{j}\left(d \sigma^{l+k p^{n}}\right)
\end{gathered}
$$

and

$$
Y\left(\psi_{n+1}^{j} \chi, \prod_{\omega \in R}\left(\zeta_{p^{n+2}}^{\sigma^{l+k p^{n}} \omega}-1\right)\right)=0
$$

Thus we have

$$
\sum_{\substack{0 \leqslant l<p^{n} \\ 0 \leqslant k<p}}\left(a_{l, k}-b_{l, k}\right) \psi_{n+1}^{j}\left(\sigma^{l+k p^{n}}\right)=0
$$


By letting $j$ run through all the integers $0<j<p^{n+1}$ with $p \nmid j$, we have a system of linear equations $A X=\mathbb{O}$, where $A$ is a $\left(p^{n+1}-p^{n}\right) \times p^{n+1}$ matrix with entries $\psi_{n+1}^{j}\left(\sigma^{l+k p^{n}}\right)$ and $X=\left(\ldots, a_{l, k}-b_{l, k}, \ldots\right)^{t}$. Since $\operatorname{rank} A=p^{n+1}-p^{n}$, the rank of solutions must be $p^{n}$. And we can exhibit $p^{n}$ independent solutions explicitly. Namely, for each $s, 0 \leqslant s<p^{n}$, let $X_{s}=\left(\ldots, f_{l, k}, \ldots\right)^{t}$ be such that

$$
f_{l, k}= \begin{cases}0 & \text { if } l \neq s \\ 1 & \text { if } l=s\end{cases}
$$

Then $X_{s}$ is a solution since $\sum_{0 \leqslant k<p} \psi_{n+1}^{j}\left(\sigma^{s+k p^{n}}\right)=0$ for all $j$, and $\left\{X_{s}\right\}_{0 \leqslant s<p^{n}}$ is clearly linearly independent. Therefore if $X=\left(\ldots, a_{l, k}-b_{l, k}, \ldots\right)^{t}$ is a solution of $A X=\mathbb{O}$, then $a_{s, k}-b_{s, k}$ is independent of $k$ for all $s, 0 \leqslant s<p^{n}$, say $a_{s, k}-b_{s, k}=e_{s}$. Then we can write $u$ as

$$
u=\prod_{l, k, \omega, \tau}\left(\zeta_{p^{n+2}}^{\sigma^{l+k p^{n}} \omega}-\zeta_{d}^{\tau}\right)^{e_{l}} \prod_{\substack{l, k, \omega \\ \nu \in \bar{\Delta}}}\left(\zeta_{p^{n+2}}^{\sigma^{l+k p^{n}} \omega}-\zeta_{d}^{\nu}\right)^{b_{l, k}} \prod_{l, k, \omega}\left(\zeta_{p^{n+2}}^{\sigma^{l+k p^{n}} \omega}-1\right)^{c_{l, k}}
$$

In this expression, the first product is in $C_{n}$, since $\prod_{k, \omega, \tau}\left(\zeta_{p^{n+2}}^{\sigma^{l+k p^{n}} \omega}-\zeta_{d}^{\tau}\right)$ is in $C_{n}$. The second and the third products in the expression, on the other hand, are circular units of $\mathbb{Q}_{n+1}$. Thus we can write $u$ as $u=v_{n} v$ for some $v_{n} \in C_{n}$ and $v \in C_{\mathbb{Q}_{n+1}}$, and $v$ satisfies $v^{\sigma^{p^{n}}}=v$. Now write $v$ as $v=\prod_{l, k, \omega}\left(\zeta_{p^{n+2}}^{\sigma^{l+k p^{n}} \omega}-1\right)^{d_{l, k}}$ and apply Ennola's Theorem to $v^{\sigma^{p^{n}}-1}=1$ with characters of the form $\psi_{n+1}^{j}, 0 \leqslant j<p^{n+1}, p \nmid j$. After a similar computation, we see that $v$ is a circular unit in $\mathbb{Q}_{n}$, hence $u \in C_{n}$. This proves (1).

$$
\text { PROOF OF (2): For each } l \geqslant 0 \text {, let } \delta_{l}=\prod_{\substack{\omega \in R \\ \tau \in \Delta}}\left(\zeta_{p^{l+1}}^{\omega}-\zeta_{d}^{\tau}\right) \text { and } \pi_{l}=\prod_{\omega \in R}\left(\zeta_{p^{l+1}}^{\omega}-1\right) .
$$

Then $\delta_{l}, \pi_{l}^{\sigma-1} \in C_{l}$. Note that for $m>n \geqslant 0$,

$$
N_{m, n}\left(\delta_{m}\right)=\prod_{\substack{\omega \in R \\ \tau \in \Delta}}\left(\zeta_{p^{n+1}}^{\omega}-\zeta_{d}^{p^{m-n} \tau}\right)=\delta_{n}
$$

since $p$ splits in $k$ and thus $\tau_{p}$ (Frobenius automorphism of $\mathbb{Q}\left(\zeta_{d}\right)$ for $p$ ) permutes $\Delta$. In particular,

$$
N_{l}\left(\delta_{l}\right)=\delta_{0}=\prod_{\omega, \tau}\left(\zeta_{p}^{\omega}-\zeta_{d}^{\tau}\right)=\frac{\prod_{\tau}\left(1-\zeta_{d}^{p \tau}\right)}{\prod_{\tau}\left(1-\zeta_{d}^{\tau}\right)}=1
$$


Also note that, for any $u \in C_{n}$, we can write $u$ as $u=u_{0} u_{1} \cdots u_{n}$, where $u_{0} \in C_{0}$ and for $k \geqslant 1, u_{k}$ is of the form

$$
u_{k}=\delta_{k} \sum_{\substack{0 \leqslant i<p^{k} \\ 0 \leqslant 1}} a_{i, j} \sigma^{i} \rho^{j}{ }_{(\sigma-1)} \sum_{0 \leqslant i<p^{k}} c_{i} \sigma^{i} .
$$

First we claim that $C_{n}=C_{0} N_{m, n} C_{m}$. Clealy $C_{0} N_{m, n} C_{m}$ is contained in $C_{n}$. To check the converse, let $u=u_{0} u_{1} \cdots u_{n}$, where $u_{k}$ is as in (**) for $k \geqslant 1$. Let $\sigma_{s}=\sigma^{p^{s}}$. Then $N_{m, k}=\sum_{0 \leqslant i<p^{m-k}} \sigma_{k}^{i}$. For each $i, 0 \leqslant i<p^{m-k}$, write $i=a p^{n-k}+b$ with $0 \leqslant a<p^{m-n}, 0 \leqslant b<p^{n-k}$. Then

$$
\begin{aligned}
N_{m, k}=\sum_{a, b} \sigma_{k}^{a p^{n-k}+b}=\left(\sum_{a} \sigma_{k}^{a p^{n-k}}\right)\left(\sum_{b} \sigma_{k}^{b}\right)=\left(\sum_{a} \sigma_{n}^{a}\right)\left(\sum_{b} \sigma_{k}^{b}\right) & =\left(\sum_{b} \sigma_{k}^{b}\right) N_{m, n}
\end{aligned}
$$

Therefore

$$
\delta_{k}=N_{m, k} \delta_{m}=N_{m, n}\left(\delta_{m}^{\sum_{b}^{b} \sigma_{k}^{b}}\right) \text { and } \pi_{k}^{\sigma-1}=N_{m, n}\left(\pi_{m}^{(\sigma-1) \sum_{b} \sigma_{k}^{b}}\right)
$$

Hence $u_{k} \in N_{m, n} C_{m}$ for each $k, 1 \leqslant k \leqslant n$.

Next we show that $C_{0} \cap N_{m, n} C_{m}=C_{0}^{p^{m-n}}$. Obviously, $C_{0}^{p^{m-n}} \subset C_{0} \cap N_{m, n} C_{m}$. For the converse, suppose $u \in C_{0} \cap N_{m, n} C_{m}$ and write $u=N_{m, n}(v)$ for some $v \in C_{m}$. As before, we can write $v=v_{0} v_{1} \cdots v_{m}$ with $v_{k}$ of the form in (**) for $k \geqslant 1$. By taking $N_{n}$, we have $N_{n}(u)=N_{n}\left(N_{m, n}(v)\right)=N_{m}(v)$. Since $N_{m}\left(v_{k}\right)=N_{k}\left(v_{k}\right)^{p^{m-k}}=1$ for $k \geqslant 1$, we obtain $u^{p^{n}}=v_{0}^{p^{m}}$. Thus $u^{-1} v_{0}^{p^{m-n}}$ is a $p^{n}$ th root of 1 in $k$, hence equals 1 . Therefore $u=v_{0}^{p^{m-n}} \in C_{0}^{p^{m-n}}$. Thus

$$
\begin{aligned}
\widehat{H}^{0}\left(G_{m, n}, C_{m}\right) & =C_{n} / N_{m, n} C_{m}=C_{0} N_{m, n} C_{m} / N_{m, n} C_{m} \\
& =C_{0} / C_{0} \cap N_{m, n} C_{m}=C_{0} / C_{0}^{p^{m-n}} .
\end{aligned}
$$

Note that $C_{0}$ is generated by $\prod_{\tau \in \Delta}\left(1-\zeta_{d}^{\tau}\right), \prod_{\tau \in \Delta}\left(1-\zeta_{d}^{\rho \tau}\right)$ and -1 . But $\prod_{\tau \in \Delta}\left(1-\zeta_{d}^{\tau}\right)$ $\prod_{\tau \in \Delta}\left(1-\zeta_{d}^{\rho \tau}\right)=1$. Hence

$$
\widehat{H}^{0}\left(G_{m, n}, C_{m}\right) \simeq \mathbb{Z} / p^{m-n} \mathbb{Z}
$$


and is generated by $\prod_{\tau \in \Delta}\left(1-\zeta_{d}^{\tau}\right)$.

Proof of (3): Let $\delta_{n}$ and $\pi_{n}$ be as in the proof of (3). We saw that $N_{n}\left(\delta_{n}\right)=$ $N_{n}\left(\pi_{n}^{\sigma-1}\right)=1$. We shall prove

$(* * *) \quad$ if $\delta_{n}^{a} \pi_{n}^{(\sigma-1) b} \in C_{n}^{\sigma-1}$, then $a \equiv b \equiv 0 \bmod p^{n}$.

This would imply $\widehat{H}^{-1}\left(G_{n}, C_{n}\right) \simeq\left(\mathbb{Z} / p^{n} \mathbb{Z}\right)^{2}$ and $\widehat{H}^{-1}\left(G_{n}, C_{n}\right)$ is generated by $\delta_{n}$ and $\pi_{n}^{\sigma-1}$ since the Herbrand quotient for $C_{n}$ is $p^{n}$ and $\hat{H}^{-1}\left(G_{n}, C_{n}\right)$ is annihilated by $p^{n}$. Then from the inflation- restriction sequence

$$
0 \rightarrow H^{1}\left(G_{n}, C_{m}^{G_{m, n}}\right) \stackrel{\text { inf }}{\longrightarrow} H^{1}\left(G_{m}, C_{m}\right) \stackrel{\text { res }}{\longrightarrow} H^{1}\left(G_{m, n}, C_{m}\right),
$$

we obtain

$$
0 \rightarrow\left(\mathbb{Z} / p^{n} \mathbb{Z}\right)^{2} \rightarrow\left(\mathbb{Z} / p^{m} \mathbb{Z}\right)^{2} \rightarrow H^{1}\left(G_{m, n}, C_{m}\right)
$$

since the first cohomology group $H^{1}$ is isomorphic to $\widehat{H}^{-1}$. Thus $\left(\mathbb{Z} / p^{m-n} \mathbb{Z}\right)^{2}$ injects into $H^{1}\left(G_{m, n}, C_{m}\right)$. But we already know that its order is $p^{2(m-n)}$. Therefore $\widehat{H}^{-1}\left(G_{m, n}, C_{m}\right) \simeq\left(\mathbb{Z} / p^{m-n} \mathbb{Z}\right)^{2}$.

It remains to show $(* * *)$. We shall prove this by induction on $n$. Suppose that $\delta_{1}^{a} \pi_{1}^{(\sigma-1) b}=u^{\sigma-1}$ for some $u \in C_{1}$. As before we can write $u=u_{0} u_{1}$, where $u_{0} \in C_{0}$ and $u_{1}$ is of the form in $(* *)$. So we have $\delta_{1}^{a} \pi_{1}^{(\sigma-1) b}=u_{1}^{\sigma-1}$, where $u_{1}=$ $\delta_{1}^{\sum a_{i, j} \sigma^{i} \rho^{j}} \pi_{1}^{(\sigma-1) \sum c_{i} \sigma^{i}}$. We apply Ennola's Theorem with the character $\psi_{1} \chi$ to this equation. Then we have

$$
a Y\left(\psi_{1} \chi, \delta_{1}\right)=\left(\psi_{1}(\sigma)-1\right)\left(\sum_{i, j} a_{i, j} \psi_{1} \chi\left(\sigma^{i} \rho^{j}\right)\right) Y\left(\psi_{1} \chi, \delta_{1}\right) .
$$

Since

$$
Y\left(\psi_{1} \chi, \delta_{1}\right)=\sum_{\omega, \tau} \psi_{1} \chi\left(-\omega d+p^{2} \tau\right)=(p-1) \frac{\phi(d)}{2} \psi_{1}(d) \neq 0
$$

we get

$$
a=\left(\psi_{1}(\sigma)-1\right)\left(\sum_{i, j} a_{i, j} \psi_{1} \chi\left(\sigma^{i} \rho^{j}\right)\right)
$$

Note that $\sum a_{i, j} \psi_{1} \chi\left(\sigma^{i} \rho^{j}\right)$ is integral. Therefore $a \equiv 0 \bmod \left(\zeta_{p}-1\right)$, hence $\bmod p$. Since $N_{1} \delta_{1}=1$,

$$
\delta_{1}^{p}=\frac{\delta_{1}^{p}}{N_{1} \delta_{1}}=\delta_{1}^{\sum_{0}^{0 \leqslant i<p}\left(1-\sigma^{i}\right)}=\delta_{1}^{\left(\sum\left(1-\sigma^{i}\right) /(\sigma-1)\right)(\sigma-1)} \in C_{1}^{\sigma-1} .
$$


Then from $\delta_{1}^{a} \pi_{1}^{(\sigma-1) b}=u^{\sigma-1}$, we obtain $\pi_{1}^{(\sigma-1) b}=v^{\sigma-1}$ for some $v \in C_{1}$. This implies that $\pi_{1}^{b}=v \alpha_{0}$ for some $\alpha_{0} \in k$. As ideals, we have $\left(\pi_{1}^{b}\right)=\left(\alpha_{0}\right)$, which is impossible unless $b \equiv 0 \bmod p$ since primes of $k$ above $p$ ramify totally in $k_{1}$.

Now we prove $(* * *)$ for $n$, assuming the result for $n-1$. Suppose $\delta_{n}^{a} \pi_{n}^{(\sigma-1) b}=$ $u_{n}^{\sigma-1}$ for some $u_{n} \in C_{n}$. By applying $N_{n, n-1}$ to both sides, we have $\delta_{n-1}^{a} \pi_{n-1}^{(\sigma-1) b}=$ $\left(N_{n, n-1} u_{n}\right)^{\sigma-1} \in C_{n-1}^{\sigma-1}$. Then by the induction hypothesis, $a \equiv b \equiv 0 \bmod p^{n-1}$. Let $a=p^{n-1} a_{1}$ and $b=p^{n-1} b_{1}$. Note that

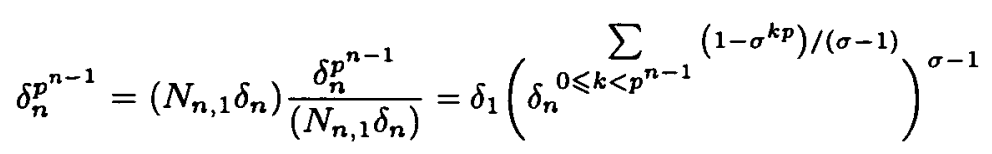

and

$$
\pi_{n}^{p^{n-1}(\sigma-1)}=\pi_{1}^{\sigma-1}\left({\pi_{n}^{k}}^{\sum\left(1-\sigma^{k p}\right)}\right)^{\sigma-1}
$$

Therefore $\delta_{n}^{a} \pi_{n}^{(\sigma-1) b}=u_{n}^{\sigma-1}$ reads $\delta_{1}^{a_{1}} \pi_{1}^{(\sigma-1) b}=v_{n}^{\sigma-1}$ for some $v_{n} \in C_{n}$. By the injectivity of the inflation map

$$
\widehat{H}^{-1}\left(G_{1}, C_{1}\right) \simeq H^{1}\left(G_{1}, C_{1}\right) \stackrel{\text { inf }}{\longrightarrow} H^{1}\left(G_{n}, C_{n}\right) \simeq \widehat{H}^{-1}\left(G_{n}, C_{n}\right),
$$

$\delta_{1}^{a_{1}} \pi_{1}^{(\sigma-1) b_{1}}$ must be in $C_{1}^{\sigma-1}$. Thus $a_{1} \equiv b_{1} \equiv 0 \bmod p$ and so $a \equiv b \equiv 0 \bmod p^{n}$. This finishes the proof.

REMARK. In the proof of (1), we did not use the splitting of $p$. So (1) is still valid even when $p$ remains inert in $k$. If $p$ remains inert in $k$, the Frobenius automorphism $\tau_{p}$ of $\mathbb{Q}\left(\zeta_{d}\right)$ for $p$ is not in $\Delta$. Thus

$$
\prod_{\tau \in \Delta}\left(1-\zeta_{d}^{\tau}\right)^{-2}=\prod_{\tau \in \Delta} \frac{1-\zeta_{d}^{p \tau}}{1-\zeta_{d}^{\tau}}=\prod_{\omega, \tau}\left(\zeta_{p}^{\omega}-\zeta_{d}^{\tau}\right)=\delta_{0}=N_{1}\left(\delta_{1}^{\tau_{p}}\right)
$$

Therefore $\prod_{\tau \in \Delta}\left(1-\zeta_{d}^{\tau}\right) \in N_{1}\left(C_{1}\right)$. With this additional information, one can modify the proof of (2) to obtain:

THEOREM 2'. Suppose $p$ remains inert in $k$. For $m>n \geqslant 0$, we have

$$
\begin{aligned}
C_{m}^{G_{m, n}} & =C_{n}, \\
\widehat{H}^{0}\left(G_{m, n}, C_{m}\right) & =\{0\}, \\
\widehat{H}^{-1}\left(G_{m, n}, C_{m}\right) & \simeq \mathbb{Z} / p^{m-n} \mathbb{Z} .
\end{aligned}
$$




\section{MAin Results}

Let $p$ be an odd prime which splits in $k$ and let $\delta_{n}=\prod_{\omega \in R, \tau \in \Delta}\left(\zeta_{p^{n+1}}^{\omega}-\zeta_{d}^{\tau}\right), \pi_{n}=$ $\prod_{\omega \in R}\left(\zeta_{p^{n+1}}^{\omega}-1\right)$ as before. We know that $\delta_{n}$ and $\pi_{n}^{\sigma-1}$ generate $\widehat{H}^{-1}\left(G_{n}, C_{n}\right)$. Let $E_{n}^{\prime}$ be the group of $p$-units of $k_{n}$.

LEMMA 1. The homomorphism $\widehat{H}^{-1}\left(G_{n}, C_{n}\right) \rightarrow \widehat{H}^{-1}\left(G_{n}, E_{n}^{\prime}\right)$ induced by the inclusion $C_{n} \rightarrow E_{n}^{\prime}$ is a zero map.

Proof: Since $G_{n}$ is cyclic, it is enough to show that $H^{1}\left(G_{n}, C_{n}\right) \rightarrow H^{1}\left(G_{n}, E_{n}^{\prime}\right)$ is a zero map. By taking limits under the inflation maps, we have a homomorphism $H^{1}\left(\Gamma, C_{\infty}\right) \longrightarrow H^{1}\left(\Gamma, E_{\infty}^{\prime}\right)$, where $C_{\infty}=\bigcup_{n \geqslant 0} C_{n}$ and $E_{\infty}^{\prime}=\bigcup_{n \geqslant 0} E_{n}^{\prime}$. By Theorem 2, $H^{1}\left(\Gamma, C_{\infty}\right) \simeq\left(\mathbb{Q}_{p} / \mathbb{Z}_{p}\right)^{2}$. On the other hand, $H^{1}\left(\Gamma, E_{\infty}^{\prime}\right)$ is a finite group [2]. Since $\left(\mathbb{Q}_{p} / \mathbb{Z}_{p}\right)^{2}$ cannot have a nontrivial finite quotient, the map $H^{1}\left(\Gamma, C_{\infty}\right) \rightarrow H^{1}\left(\Gamma, E_{\infty}^{\prime}\right)$ is a zero map. Then the lemma follows from the injectivity of the inflation maps.

Thus $\delta_{n}=\alpha_{n}^{\sigma-1}$ for some $p$-unit $\alpha_{n}$ in $k_{n}$ by the lemma. Let $\wp_{n}$ and $\widetilde{\wp}_{n}$ be the prime ideals of $k_{n}$ above $p$ as in the introduction. Then $\left(\alpha_{n}\right)=\wp_{n}^{g_{n}} \widetilde{\wp}_{n} \widetilde{g}_{n}$ for some integers $g_{n}$ and $\widetilde{g}_{n}$. If $\delta_{n}=\alpha_{n}^{\sigma-1}=\beta_{n}^{\sigma-1}$ for some other $p$-unit $\beta_{n}$, then $\alpha_{n}=\beta_{n} \alpha_{0}$ for some $p$-unit $\alpha_{0} \in k_{0}$. Thus $g_{n}$ and $\tilde{g}_{n}$ are determined uniquely modulo $p^{n}$ by $\delta_{n}$ since $\wp_{0}$ and $\widetilde{\wp_{0}}$ ramify totally in $k_{n}$. If $\delta_{m}=\alpha_{m}^{\sigma-1}$ with $\left(\alpha_{m}\right)=\wp_{m}^{g_{m}}{\widetilde{\wp_{m}}}^{\widetilde{g}_{m}}$ for $m>n$, then $\delta_{n}=N_{m, n} \delta_{m}=\left(N_{m, n} \alpha_{m}\right)^{\sigma-1}$ and $\left(N_{m, n} \alpha_{m}\right)=\wp_{n}^{g_{m}} \widetilde{\wp_{n}} \widetilde{g}_{m}$. Therefore $g_{m} \equiv g_{n}, \tilde{g}_{m} \equiv \tilde{g}_{n} \bmod p^{n}$.

THEOREM 3. Let $\delta_{n}=\alpha_{n}^{\sigma-1}$ with $\left(\alpha_{n}\right)=\wp_{n}^{g_{n}}{\widetilde{\wp_{n}}}_{\tilde{g}_{n}}$. Then $g_{n}-\widetilde{g}_{n} \equiv g_{1}-\widetilde{g}_{1} \equiv$ $\pm \sqrt{d} B_{1, \chi \omega^{-1}} \bmod p \mathbb{Z}_{p}$.

REMARK. The signature in the theorem depends on the embedding of $\overline{\mathbb{Q}}$ into $\overline{\mathbb{Q}_{p}}$. Fix an embedding $\iota$ once and for all and assume that under this embedding, $k_{n}$ is completed at $\wp_{n}$ rather than $\widetilde{\wp_{n}}$. We denote $\iota\left(\zeta_{d}\right)$ just by $\zeta_{d}$. Let $p(\tau)$ be the integer modulo $d$ corresponding to $\tau$ under the isomorphism $\bar{\Delta} \simeq(\mathbb{Z} / d \mathbb{Z})^{\times}$. Then $\iota\left(\zeta_{d}^{\tau}\right)=\iota\left(\zeta_{d}^{p(\tau)}\right)=\iota\left(\zeta_{d}\right)^{p(\tau)}=\zeta_{d}^{p(\tau)}$. Again we simply write $\zeta_{d}^{\tau}$ for $\iota\left(\zeta_{d}^{\tau}\right)=\zeta_{d}^{p(\tau)}$ in $\overline{\mathbb{Q}_{p}}$.

Before we prove Theorem 3, we need the following proposition which is valid even when $p$ remains inert in $k$.

PROPOSITION 1 .

$\sum_{\substack{\omega \in R \\ \tau \in \bar{\Delta}}} \chi(\tau) \log _{p}\left(\zeta_{p^{2}}^{\omega}-\zeta_{d}^{\tau}\right) \equiv-\chi(p) \sqrt{d} B_{1, \chi \omega^{-1}} \bmod \left(\zeta_{p^{2}}-1\right)^{p-1}$. 
Proof: For $0 \leqslant i<p, 0 \leqslant k<p$, let

$$
\begin{aligned}
& T_{i}=\sum_{\omega \in R, \tau \in \bar{\Delta}} \chi(\tau) \log _{p}\left(\zeta_{p^{2}}^{\sigma^{i} \omega}-\zeta_{d}^{\tau}\right), \\
& S_{k}=\sum_{0 \leqslant i<p} \psi^{k}\left(\sigma^{i}\right) t_{I},
\end{aligned}
$$

where $\psi=\psi_{1}$. When $k=0$,

$$
\begin{aligned}
S_{0} & =\sum_{0 \leqslant i<p} T_{i} \\
& =\sum_{\tau \in \bar{\Delta}} \chi(\tau) \sum_{\substack{0 \leqslant i<p \\
\omega \in R}} \log _{p}\left(\zeta_{p^{2}}^{\sigma^{i} \omega}-\zeta_{d}^{\tau}\right) \\
& =\sum_{\tau \in \bar{\Delta}} \chi(\tau) \log _{p} \frac{1-\zeta_{d}^{p^{2} \tau}}{1-\zeta_{d}^{p \tau}} \\
& =\left(\chi\left(p^{-2}\right)-\chi\left(p^{-1}\right)\right) \sum_{\tau \in \bar{\Delta}} \chi(\tau) \log _{p}\left(1-\zeta_{d}^{\tau}\right) \\
& =(1-\chi(p)) \sum_{\substack{a \\
(a, d)=1}} \chi(a) \log _{p}\left(1-\zeta_{d}^{a}\right) \\
& =-\frac{1-\chi(p)}{p-\chi(p)} p \sqrt{d} L_{p}(1, \chi) .
\end{aligned}
$$

When $k \neq 0$,

$$
\begin{aligned}
S_{k} & =\sum_{i, \omega, \tau} \psi^{k} \chi\left(\sigma^{i} \tau\right) \log _{p}\left(\zeta_{p^{2}}^{\sigma^{i} \omega}-\zeta_{d}^{\tau}\right) \\
& =\overline{\psi^{k}}(d) \sum_{1 \leqslant b \leqslant p^{2} d} \psi^{k} \chi(b) \log _{p}\left(1-\zeta_{p^{2} d}^{b}\right) \\
& =-\frac{\overline{\psi^{k}}(d) p^{2} d}{\tau\left(\overline{\psi^{k}} \chi\right)} L_{p}\left(1, \overline{\psi^{k}} \chi\right)
\end{aligned}
$$

where $\tau\left(\overline{\psi^{k}} \chi\right)$ is the Gauss sum of the character $\overline{\psi^{k}} \chi$. Note that

$$
\begin{aligned}
& \tau\left(\overline{\psi^{k}} \chi\right)=\sum_{1 \leqslant a<p^{2} d} \overline{\psi^{k}} \chi(a) \zeta_{p^{2} d}^{a} \\
& =\sum_{\substack{0 \leqslant x<d \\
0 \leqslant y<p^{2}}} \overline{\psi^{k}} \chi\left(p^{2} x+d y\right) \zeta_{p^{2} d}^{p^{2} x+d y}
\end{aligned}
$$




$$
\begin{aligned}
& =\overline{\psi^{k}}(d)\left(\sum_{y} \overline{\psi^{k}}(y) \zeta_{p^{2}}^{y}\right)\left(\sum_{x} \chi(x) \zeta_{d}^{x}\right) \\
& =\overline{\psi^{k}}(d) \tau\left(\overline{\psi^{k}}\right) \tau(\chi)
\end{aligned}
$$

We also have

$$
\begin{aligned}
& \tau\left(\overline{\psi^{k}}\right)=\sum_{0 \leqslant y<p^{2}} \overline{\psi^{k}}(y) \zeta_{p^{2}}^{y} \\
& =\sum_{\substack{0 \leqslant i<p \\
\omega \in R}} \overline{\psi^{k}}\left(\sigma^{i} \omega\right) \zeta_{p^{2}}^{\sigma^{i} \omega} \\
& =\sum_{i, \omega} \zeta_{p}^{-k i} \zeta_{p^{2}}^{(1+i p) \omega} \\
& =\sum_{\omega} \zeta_{p^{2}}^{\omega}\left(\sum_{i} \zeta_{p}^{(\omega-k) i}\right) \\
& =p \zeta_{p^{2}}^{\omega(k)},
\end{aligned}
$$

where $\omega(k)$ is the root of 1 in $\mathbb{Z}_{p}$ satisfying $\omega(k) \equiv k \bmod p$. Therefore, for $k \neq 0$,

$$
S_{k}=-p \sqrt{d} \zeta_{p^{2}}^{-\omega(k)} L_{p}\left(1, \overline{\psi^{k}} \chi\right)
$$

Thus we have a system of linear equations

$$
\left(\psi^{k}\left(\sigma^{i}\right)\right)\left(\begin{array}{c}
T_{0} \\
T_{1} \\
\vdots \\
T_{p-1}
\end{array}\right)=-p \sqrt{d}\left(\begin{array}{c}
\frac{1-\chi(p)}{p-\chi(p)} L_{p}(1, \chi) \\
\vdots \\
\zeta_{p^{2}}^{-\omega(k)} L_{p}\left(1, \overline{\psi^{k}} \chi\right) \\
\vdots
\end{array}\right)
$$

By solving this equation, we have

$$
T_{0}=-\sqrt{d}\left(\frac{1-\chi(p)}{p-\chi(p)} L_{p}(1, \chi)+\sum_{1 \leqslant k \leqslant p-1} \zeta_{p^{2}}^{-\omega(k)} L_{p}\left(1, \overline{\psi^{k}} \chi\right)\right)
$$

Since $L_{p}\left(1, \overline{\psi^{k}} \chi\right) \equiv L_{p}(1, \chi) \equiv L_{p}(0, \chi)=-B_{1, \chi \omega^{-1}} \bmod \zeta_{p}-1$

$$
T_{0} \equiv \sqrt{d}\left(1-\chi(p)+\sum_{1 \leqslant k \leqslant p-1} \zeta_{p^{2}}^{\omega(k)}\right) B_{1, \chi \omega^{-1}} \bmod \zeta_{p}-1
$$


Since $\zeta_{p^{2}}^{\omega(k)} \equiv \zeta_{p^{2}}^{k} \bmod \left(\zeta_{p}-1\right), 1+\sum_{k} \zeta_{p^{2}}^{\omega(k)} \equiv\left(1-\zeta_{p}\right) /\left(1-\zeta_{p^{2}}\right) \equiv 0 \bmod \left(1-\zeta_{p^{2}}\right)^{p-1}$ Therefore $T_{0} \equiv-\chi(p) \sqrt{d} B_{1, \chi \omega^{-1}} \bmod \left(\zeta_{p^{2}}-1\right)^{p-1}$.

Proof of Theorem 3: We already know that $g_{n} \equiv g_{1}$ and $\tilde{g}_{n} \equiv \tilde{g}_{1} \bmod p$. For brevity, we denote $g_{1}$ and $\widetilde{g}_{1}$ by $g$ and $\tilde{g}$. We read $\delta_{1}=\alpha_{1}^{\sigma-1}$ in $\overline{\mathbb{Q}_{p}}$ under the embedding $\iota$. Since $k_{1}$ is assumed to be completed at $\wp_{1}$, we have $\left(\alpha_{1}\right)=\left(\pi_{1}\right)^{g}$. Let $\pi=\zeta_{p^{2}}-1$. Then $\left(\alpha_{1}\right)=(\pi)^{g(p-1)}$ in $\mathbb{Q}_{p}\left(\zeta_{p^{2}}\right)$. By taking $\rho \in \Delta_{k}$, we get $\delta_{1}^{\rho}=\alpha_{1}^{\rho(\sigma-1)}$ and $\left(\alpha_{1}^{\rho}\right)=\wp_{1}^{\widetilde{g} \wp_{1}^{g}}$ in $k_{1}$, hence $\left(\alpha_{1}^{\rho}\right)=(\pi)^{\widetilde{g}(p-1)}$ in $\mathbb{Q}_{p}\left(\zeta_{p^{2}}\right)$. Thus $\delta_{1}^{1-\rho}=\alpha_{1}^{(1-\rho)(\sigma-1)}$ and $\left(\alpha_{1}^{1-\rho}\right)=(\pi)^{(g-\tilde{g})(p-1)}$. Hence, in $\mathbb{Q}_{p}\left(\zeta_{p^{2}}\right)$,

$$
\delta_{1}^{1-\rho}=\pi^{(g-\tilde{g})(p-1)(\sigma-1)} \eta^{\sigma-1}
$$

for some unit $\eta$ in $\mathbb{Q}_{p}\left(\zeta_{p^{2}}\right)$. It is easy to see that

$$
\pi^{\sigma-1} \equiv 1+\pi^{p-1} \bmod \pi^{p}, \text { and } \eta^{\sigma-1} \equiv 1 \bmod \pi^{p} .
$$

Therefore

$$
\delta_{1}^{1-\rho} \equiv 1+(g-\tilde{g})(p-1) \pi^{p-1} \equiv 1+(\widetilde{g}-g) \pi^{p-1} \bmod \left(\zeta_{p}-1\right) .
$$

Hence

$$
\log _{p} \delta_{1}^{1-\rho} \equiv \log _{p}\left(1+(\tilde{g}-g) \pi^{p-1}\right) \bmod \left(\zeta_{p}-1\right)
$$

Now we compute both sides of this congruence.

$$
\begin{aligned}
\text { LHS } & =\log _{p} \delta_{1}^{1-\rho} \\
& =\log _{p} \prod_{\substack{\omega \in R \\
\tau \in \Delta}}\left(\zeta_{p^{2}}^{\omega}-\zeta_{d}^{\tau}\right)^{1-\rho} \\
& =\log _{p} \prod_{\substack{\omega \in R \\
\tau \in \bar{\Delta}}}\left(\zeta_{p^{2}}^{\omega}-\zeta_{d}^{\tau}\right)^{\chi(\tau)} \\
& =\sum_{\omega \in R} \chi(\tau) \log _{p}\left(\zeta_{p^{2}}^{\omega}-\zeta_{d}^{\tau}\right) \\
& \equiv-\sqrt{d} B_{1, \chi \omega^{-1}} \bmod \pi .
\end{aligned}
$$

On the other hand,

$$
\begin{aligned}
\text { RHS } & =\log _{p}\left(1+(\tilde{g}-g) \pi^{p-1}\right) \\
& \equiv(\widetilde{g}-g) \pi^{p-1}-\frac{1}{2}\left((\tilde{g}-g) \pi^{p-1}\right)^{2}+\ldots+\frac{1}{p}\left((\tilde{g}-g) \pi^{p-1}\right)^{p}-\ldots
\end{aligned}
$$


In this expression, every term except $\left((\tilde{g}-g) \pi^{p-1}\right)^{p} / p$ is congruent to $0 \bmod \pi$, and $\pi^{(p-1) p} / p \equiv-1 \bmod \pi$. Therefore

$$
\log _{p}\left(1+(\tilde{g}-g) \pi^{p-1}\right) \equiv g-\tilde{g} \bmod \pi .
$$

By equating both sides, we obtain

$$
g-\tilde{g} \equiv-\sqrt{d} B_{1, \chi \omega^{-1}} \bmod \pi .
$$

Since both sides are in $\mathbb{Z}_{p}$, the congruence holds $\bmod p \mathbb{Z}_{p}$.

THEOREM 4. Suppose an odd prime $p$ splits in $k=\mathbb{Q}(\sqrt{m})$. If $p \mid B_{1, \chi \omega^{-1}}$, then $p \mid h_{n}$ for all $n \geqslant 1$.

Proof: By class field theory, it is enough to show that $p \mid h_{1}$. If $p \mid h_{0}$, then there is nothing to prove. So we assume that $p \nmid h_{0}$. In particular, there is no nontrivial capitulation from $k_{0}$ to $k_{1}$.

Let $\delta_{1}=\alpha_{1}^{\sigma-1}$ and $\left(\alpha_{1}\right)=\wp_{1}^{g_{1}}{\widetilde{\wp_{1}}}^{g_{1}}$ as before. Since $p \mid B_{1, \chi \omega^{-1}}, g_{1} \equiv \widetilde{g}_{1} \bmod p$ by Theorem 3. Let $g$ be such that $0 \leqslant g<p$ and $g_{1} \equiv \widetilde{g}_{1} \equiv g \bmod p$. Then

$$
\left(\alpha_{1}\right)=\left(\wp_{1} \widetilde{\wp_{1}}\right)^{g} I_{0}=\left(\pi_{1}^{g}\right) I_{0}
$$

for some ideal $I_{0}$ of $k_{0}$. Since there is no nontrivial capitulation, $I_{0}=\left(\alpha_{0}\right)$ for some $\alpha_{0} \in k_{0}$. Hence $\left(\alpha_{1}\right)=\left(\pi_{1}^{g} \alpha_{0}\right)$ and $\delta_{1}=\pi_{1}^{g(\sigma-1)} \eta_{1}^{\sigma-1}$ for some $\eta_{1} \in E_{1}$. Thus $H^{1}\left(G_{1}, C_{1}\right) \rightarrow H^{1}\left(G_{1}, E_{1}\right)$ is not injective. From the short exact sequence $0 \rightarrow C_{1} \rightarrow$ $E_{1} \rightarrow E_{1} / C_{1} \rightarrow 0$, we get a long exact sequence

$$
0 \rightarrow C_{0} \rightarrow E_{0} \rightarrow\left(E_{1} / C_{1}\right)^{G_{1}} \rightarrow H^{1}\left(G_{1}, C_{1}\right) \rightarrow H^{1}\left(G_{1}, E_{1}\right) \rightarrow .
$$

Therefore $\left(E_{1} / C_{1}\right)^{G_{1}} \otimes \mathbb{Z}_{p} \neq\{0\}$. Hence $p \mid\left[E_{1}: C_{1}\right]$ and so $p \mid h_{1}$ by the index theorem.

Corollary. Let $M_{\infty}$ and $L_{\infty}$ be as in the introduction. If $\mathrm{Gal}\left(M_{\infty} / k_{\infty}\right)$ is nontrivial, then $\mathrm{Gal}\left(L_{\infty} / k_{\infty}\right)$ is also nontrivial.

PRoOF: As in the proof of Theorem 1, if $\mathrm{Gal}\left(M_{\infty} / k_{\infty}\right)$ is nontrivial, then $f_{\chi}$ is not a unit in $\Lambda$. Hence $f_{\chi}(0)=-B_{1, \chi \omega^{-1}}$ is divisible by $p$. Thus the corollary follows from Theorem 4.

\section{REFERENCES}

[1] V. Ennola, 'On relations between cyclotomic units', J. Number Theory 4 (1972), 236-247.

[2] K. Iwasawa, 'On cohomology groups of units for $\mathbb{Z}_{p}$-extensions', Amer. J. Math. 105 (1983), 189-200. 
[3] S. Lang, Cyclotomic fields, Graduate Texts in Mathematics I and II 59, 69 (Springer-Verlag, Berlin, Heidelberg, New York, 1990).

[4] B. Mazur and A. Wiles, 'Class fields of abelian extensions of $\mathbb{Q}$ ', Invent. Math 76 (1984), 179-330.

[5] A. Scholz, 'Über die Beziehung der Klassenzahlen quadratischer Körper zueinander', $J$. Reine Angew Math. 166 (1932), 201-203.

[6] W. Sinnott, 'On the Stickelberger ideal and the circular units of an abelian field', Invent. Math. 62 (1980), 181-234.

[7] L. Washington, Introduction to cyclotomic fields, Graduate Texts in Mathematics 83 (Springer-Verlag, Berlin, Heidelberg, New York, 1980).

Department of Mathematics

Inha University

Inchon

Korea

e-mail: jmkim@math.inha.ac.kr 